\title{
Las pequeñas y medianas empresas del sector metalúrgico en la zona sur de la Comunidad de Madrid: Gestión medioambiental y necesidades de
} formación ${ }^{(\cdot)}$

\author{
A. Ureña*, J. Rams*, F.J. Méndez* y J. Rodríguez \\ Resumen En este trabajo se presentan los resultados de un análisis específico de la gestión \\ medioambiental y las necesidades de formación de las pequeñas y medianas empresas \\ (PYMES) del sector metalúrgico de la zona sur de la Comunidad de Madrid. Los datos más \\ significativos se han obtenido a través de una encuesta telefónica realizada a más de 170 \\ empresas de la zona. La mayoría de los empleados de las empresas encuestadas no \\ consideran necesaria una formación específica sobre impacto medioambiental. No \\ obstante, el estudio indica el interés, entre otros temas, por el control de vertidos y residuos, \\ la implantación de sistemas de gestión medioambiental y un mayor conocimiento de la \\ legislación y normativa aplicable al sector.
}

Palabras clave Industria metalúrgica. Medioambiente. Madrid.

\section{Environmental management and educational needs of the small and medium-sized businesses of the metallurgical sector in the south region of Madrid}

\begin{abstract}
In this study, the environmental management and needs of the small and medium-sized businesses of the metallurgical industry in the south region of Madrid were analysed. Information was obtained through a telephone questionnaire distributed to more than 170 companies. Although most of the respondent employees do not consider environmental training one of their priorities, they expressed their interest in implementing Environmental Management Systems, waste minimization and a higher knowledge of the specific legal aspects.
\end{abstract}

Keywords Metallurgical sector. Environmental problems. Madrid.

\section{INTRODUCCIÓN}

En la actualidad, el respeto al medio ambiente se ha convertido en una preocupación social que se ha plasmado en una normativa cada vez más exigente con la gestión empresarial. En consecuencia, las empresas se han visto obligadas a introducir cambios tecnológicos importantes en los procesos productivos y en los modelos de gestión. La formación de los trabajadores en aspectos medioambientales, es crítica para dirigir adecuadamente los cambios estructurales y de organización que las empresas necesitan para no descolgarse en este proceso. En las regiones industrialmente fuertes es imprescindible realizar un esfuerzo de sensibilización y formación dirigido a los trabajadores de aquellas empresas cuya actividad incida en el medio ambiente. Conocer el grado de implantación de los sistemas de gestión medioambiental y de las técnicas de producción limpia, su efectividad y la satisfacción que proporcionan, es el objetivo de numerosos estudios medioambientales centrados en una región determinada ${ }^{[1]}$.

En esta línea, la Fundación Biodiversidad y la Universidad Rey Juan Carlos colaboran en un proyecto por el desarrollo de actuaciones de formación y sensibilización, análisis de necesidades formativas y creación de estructuras en el marco del Programa Operativo "Iniciativa Empresarial y Formación Continua" del Fondo Social Europeo. Dicho proyecto se dirige a empleados de PYMES y autónomos ubicados en las zonas de influencia de

(•) Trabajo recibido el día 17 de junio de 2003 y aceptado en su forma final el día 25 de marzo de 2004.

(*) Escuela Superior de Ciencias Experimentales y Tecnología, Universidad Rey Juan Carlos. c/ Tulipán s/n, 28933 Móstoles, Madrid. 
Las pequeñas y medianas empresas del sector metalúrgico en la zona sur de la Comunidad de Madrid: Gestión medioambiental y necesidades de formación

A. UREÑA, J. RAms, F.J. MÉNDEz y J. RODRíGUEZ

la Universidad ${ }^{[2]}$. En este trabajo se resumen los resultados de un análisis específico dedicado al sector del metal ubicado en la zona sur de Madrid ${ }^{[3]}$. Esta región, formada por el eje que constituyen, principalmente, los municipios de Móstoles, Alcorcón, Fuenlabrada, Getafe y Leganés, es la mayor de las áreas productivas de la región. En ella se sitúan más de treinta mil PYMES que contribuyen de una manera significativa al empleo industrial de la Comunidad de Madrid. El porcentaje de las empresas dedicadas al sector del metal es del 3,5\%. Aproximadamente el $50 \%$ de estas empresas pueden considerarse micropymes, al tener plantillas de menos de 10 empleados. En lo que respecta al tiempo de implantación, alrededor del $70 \%$ tienen menos de 20 años, si bien se aprecia un crecimiento lento en los dos últimos años, ya que solamente se han creado empresas en el sector de la calderería. El mayor número de empresas se sitúa en el subsector del mecanizado, mientras que el número menor corresponde al de la fundición, aunque con un mayor número de empleados por empresa.

La problemática ambiental asociada a la industria del metal es muy variada como consecuencia del gran número de procesos productivos diferentes aplicados en los sectores de fundición, conformado y mecanizado, tratamiento de superficies y procesos de unión. Sin entrar en detalles que pueden consultarse en otros lugares ${ }^{[45}$, puede decirse que el impacto medioambiental asociado a la industria metalúrgica incluye, al menos, la generación de residuos sólidos, polución del aire, aguas residuales, consumo de energía, contaminación del suelo y ruido. Existen numerosas soluciones técnicas que reducen estos problemas sin apenas modificar el coste del producto. La implantación de sistemas de gestión medioambiental en las empresas contribuiría a reducir la contaminación ambiental organizando la producción de la manera más adecuada.

En el estudio se analizan datos suministrados por empleados de las PYMES de la industria del metal de la zona sur de la Comunidad de Madrid con los siguientes objetivos:

- Conocer el grado de sensibilización medioambiental de la industria del metal.

- Determinar el grado de implantación de sistemas de gestión medioambiental y medidas de producción limpia, sus resultados y el grado de satisfacción alcanzado.

- Determinar las necesidades de formación medioambiental de la industria metalúrgica y las vías preferidas para recibir la información.

\section{METODOLOGÍA}

Los datos más significativos se obtuvieron a partir de una investigación cuantitativa, realizada por medio de una encuesta basada en un cuestionario, sobre una base censal formada por un total de 880 pequeñas y medianas empresas (PYMES). Las entrevistas se realizaron telefónicamente, utilizando un sistema CATI (Computer Aided Telephone Interviewing), durante el mes de septiembre de 2002 . La selección de las empresas a entrevistar se efectuó de manera aleatoria sobre los listados telefónicos disponibles (Dun \& Bradstreet). El número de empresas que respondieron a la encuesta fue un total de 174 , lo que supone un 19,8 \% del total de la base censal empleada. Este tamaño muestral correspondería a un error muestral teórico máximo en la determinación de una proporción $(\mathrm{p}=\mathrm{q}=50 \%)$, de $\pm 4 \%$.

El grupo de empresas del sector metalúrgico que contestaron a las encuestas se subdividió por el tipo de actividades primarias que realizaban (fundición, mecanizado, recubrimiento, calderería y conformado). Dado que el grupo de empresas pertenecientes al subsector de fundición de metales que participó en la encuesta era inferior al error muestral, este grupo no se consideró de forma diferenciada.

\section{RESULTADOS Y DISCUSIÓN}

\subsection{Grado de sensibilización en las PYMES}

El primer paso para analizar las necesidades y problemas de las PYMES del metal en relación con el medio ambiente es conocer lo que los empleados opinan sobre el impacto ambiental de sus propias empresas. En este sentido, la encuesta proporciona datos ciertamente curiosos que se han recogido en la figura 1 . Aunque casi un $70 \%$ de los encuestados dice estar muy o bastante preocupado por las cuestiones medioambientales relacionadas con su actividad, la inmensa mayoría considera que el impacto ambiental de su empresa es bajo o nulo. Esta respuesta indica falta de conocimiento de los verdaderos problemas que cada empresa causa o bien, una escasa sensibilización sobre la importancia de reducir tanto el consumo de recursos naturales limitados como la contaminación producida. Cabe resaltar que las empresas que se consideran más agresivas y también más preocupadas son las que tienen un mayor número de empleados y, en general, una mayor antigüedad y tradición en su sector. 

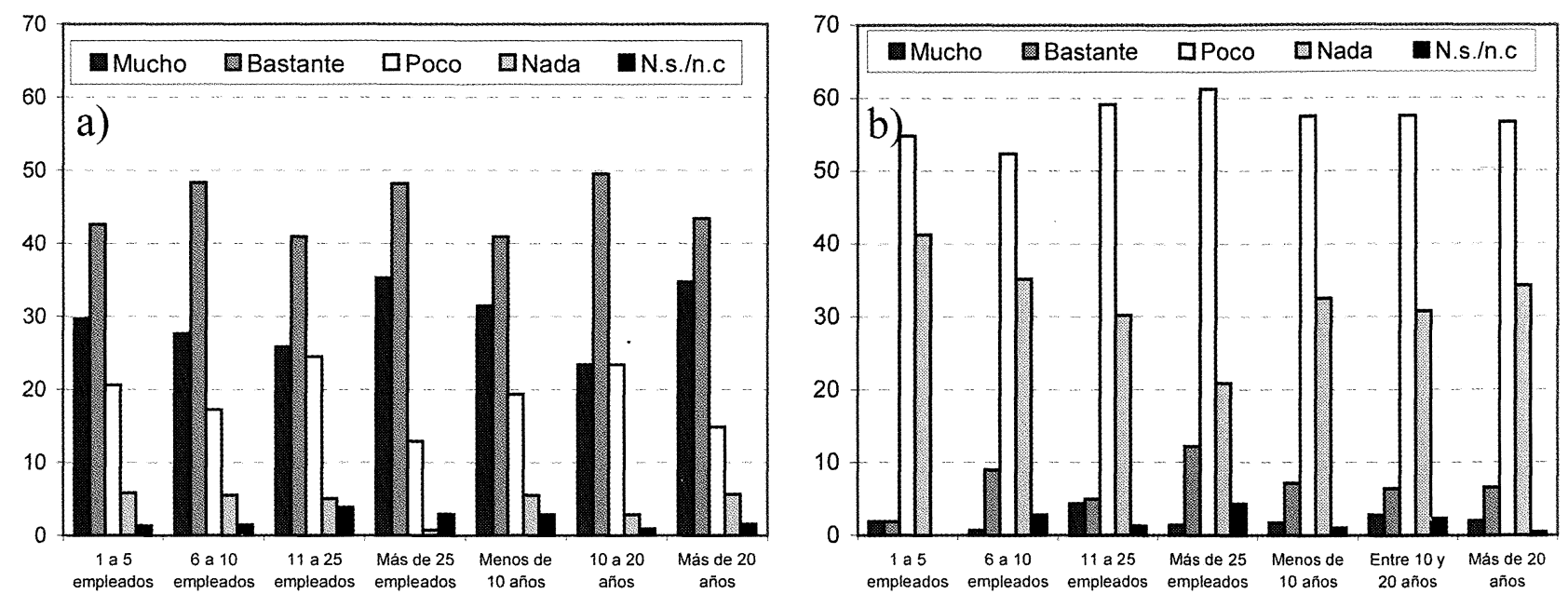

Figura 1. Grado de preocupación (a) y de estimación de su impacto ambiental (b) de las empresas.

Figure 1. Wariness (a) and estimation of their environmental impact (b) of metal industries.

\subsection{Implantación de sistemas de gestión am- biental}

Una gran parte de los problemas ambientales tienen su origen en la organización de la empresa. Con la implantación de un sistema de gestión medioambiental se consigue desarrollar, con un mayor grado de eficacia, la política ambiental de la empresa gracias, entre otras cosas, al control y registro de las actuaciones.

Los datos de la encuesta a este respecto son muy claros: poco más de la mitad de las industrias metalúrgicas conoce lo que es un sistema de gestión ambiental. Entre los que responden afirmativamente, el grado de conocimiento de metodologías concretas como la norma ISO $14001{ }^{[6]}$ y el sistema de gestión europeo EMAS [7], es mucho más reducido, como se desglosa en la figura 2 . La

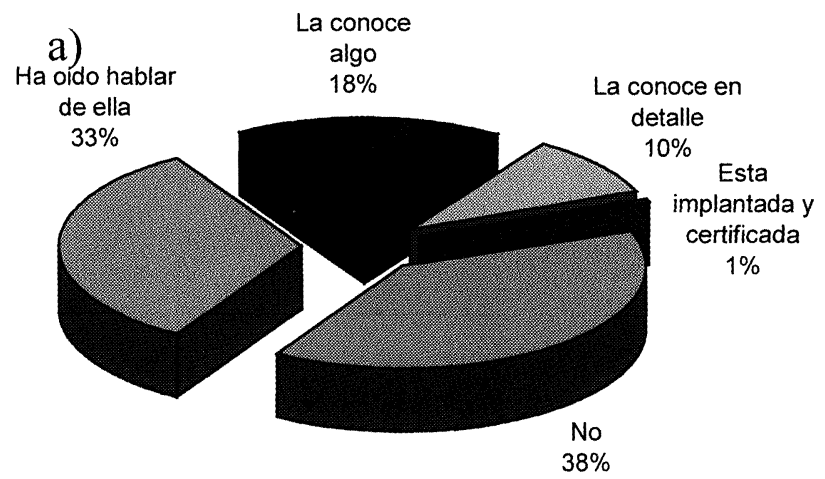

figura 3 muestra que la implantación de sistemas de gestión ambiental es muy reducida en todos los subsectores con la única excepción del de recubrimientos, donde más de un $80 \%$ de las empresas lo conocen, casi un $40 \%$ de las empresas lo tienen implantado y en un $25 \%$ de los casos llevan más de 3 años con él.

¿Cuál es el motivo de esta falta de interés? Además del desconocimiento de los sistemas de gestión medioambiental, ya puesto de manifiesto, las razones aducidas por las empresas son el temor a que se produzca un aumento de los costes, del personal necesariơ $y$, en definitiva, una pérdida de eficacia. Por el contrario, las respuestas de las empresas que lo tienen implantado indican que tras la implantación no sólo se cumple la normativa medioambiental y se reduce el impacto ambiental de la industria, sino que se reducen los costes y se

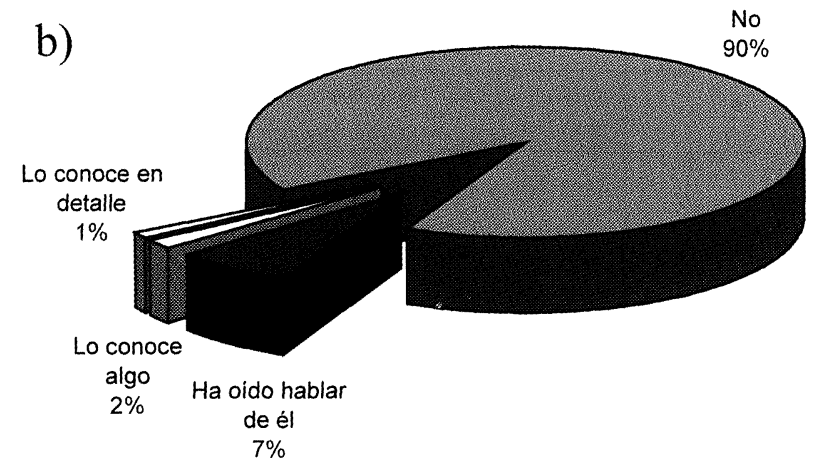

Figura 2. Grado de conocimiento de la norma ISO 14001 (a) y del sistema de gestión EMAS (b).

Figure 2. Knowledge of ISO 14001 (a) and EMAS environmental management system (b). 
Las pequeñas y medianas empresas del sector metalúrgico en la zona sur de la Comunidad de Madrid: Gestión medioambiental y necesidades de formación

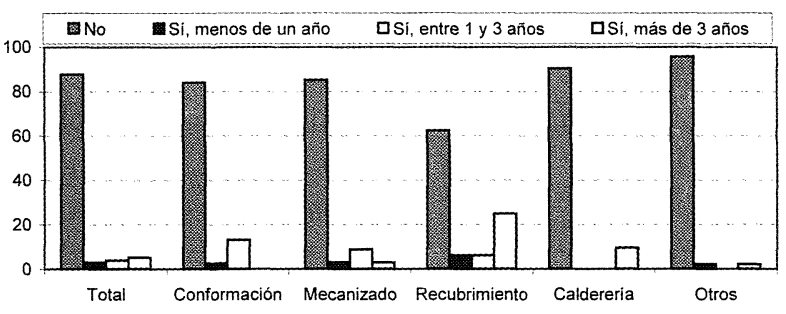

Figura 3. Grado de implantación de los șistemas de gestión ambiental y su antigüedad.

Figure 3. Implantation degree of environmental management systems according to their age.

obtienen otros beneficios empresariales como, por ejemplo, una mayor satisfacción de los clientes (figura 4). Estos resultados se producen en, prácticamente, todos los subsectores metalúrgicos pero destaca el caso de las industrias de conformado y de calderería. En definitiva, las ventajas de la implantación de un sistema de gestión medioambiental son casi indiscutibles. Si las empresas no trabajan en esa dirección es, básicamente, por desconocimiento de los beneficios de todo tipo que pueden obtener. Esto abre la discusión hacia las necesidades de formación en gestión medioambiental.

\subsection{Necesidades de formación}

A pesar de los resultados presentados anteriormente, las empresas encuestadas consideran de forma mayoritaria (más del $60 \%$ ) que no es necesaria una formación específica en temas medioambientales y sólo el $8 \%$ realiza actuaciones de formación (Fig. 5a). Esta situación es extensible a todos los subsectores, exceptuando el grupo de empresas del subsector de recubrimientos, que cree, en una proporción ligera- mente superior al $50 \%$, que este tipo de formación es de interés para las actividades productivas que realiza su empresa. De hecho, son los empleados de estas PYMES los que actualmente más acciones formativas en temas medioambientales siguen, siendo una proporción significativa (superior al $10 \%)$ los que lo hacen de una forma regular.

La figura $5 b$ ) resume el interés de los encuestados por la formación medioambiental. La mayoría resalta las necesidades formativas en el control de vertidos y residuos, una de las mayores causas de impacto medioambiental del sector del metal. También aparecen como temas prioritarios los relacionados con la formación en sistemas de gestión medioambiental y los específicos de legislación. De nuevo destaca el interés en aspectos legislativos mostrado por los trabajadores de las empresas que centran su actividad primaria en el recubrimiento de componentes metálicos (más del $60 \%$ ), íntimamente relacionado con la mayor presión normativa a la que están sometidas este tipo de actividades.

La limitada sensibilización en aspectos medioambientales de las PYMES del sector metalúrgico de la zona sur de la Comunidad de Madrid queda reflejada al comprobar que sólo un $40 \%$ de ellas está interesada en recibir formación gratuita en las diferentes temáticas antes enumeradas. Sólo el subsector de recubrimientos demuestra un interés mayoritario (cerca del $70 \%$ ) en aprovechar estas oportunidades formativas. Probablemente, el reducido tamaño de las empresas es uno de los factores limitantes a la hora de asistir a cursos presenciales. Afortunadamente, la mayoría de las PYMES metalúrgicas disponen, en los lugares de trabajo, de las nuevas tecnologías de la información y de la comunicación (el $75 \%$ tiene acceso a
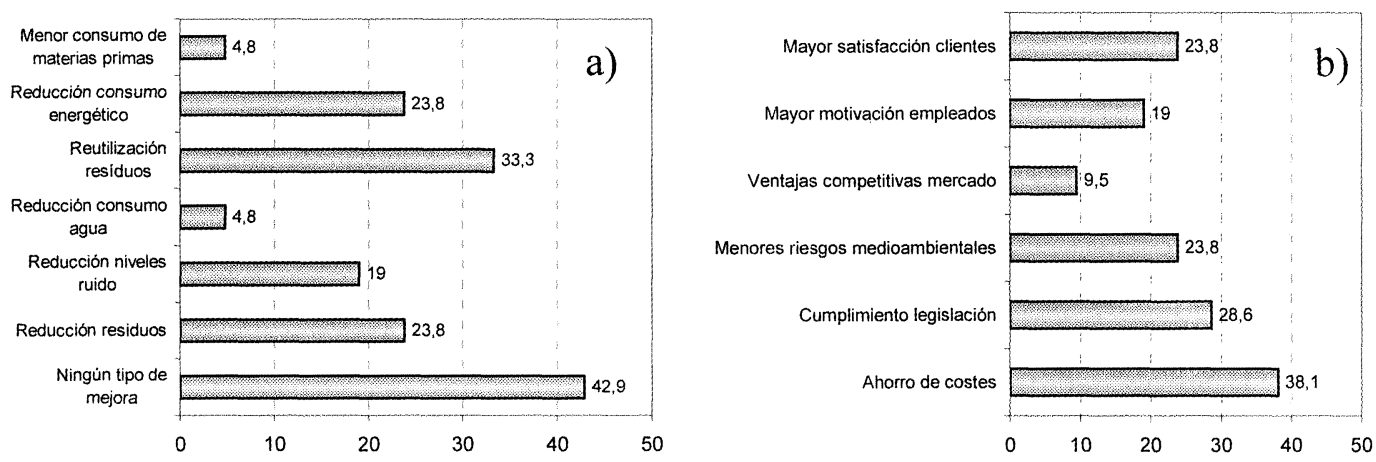

Figura 4. Beneficios medioambientales (a) y empresariales (b) obtenidos tras implantar algún sistema de gestión medioambiental.

Figure 4. Environmental (a) and business benefits (b) obtained with environmental management systems. 


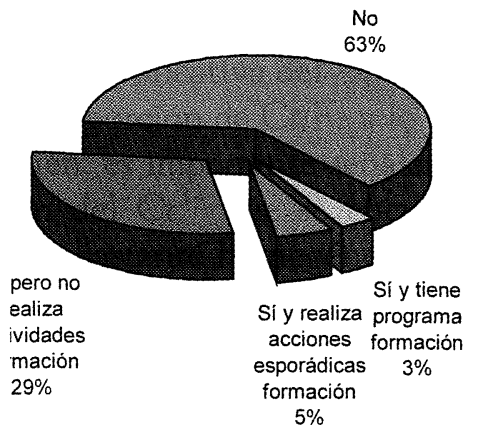

$5 \%$

a)

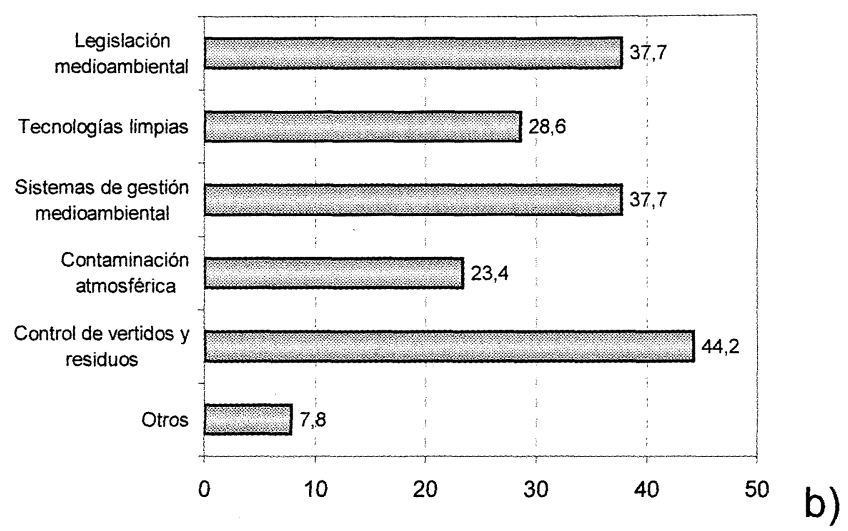

Figura 5. Consideración de la necesidad de formación y su forma de aplicación (a) y necesidades formativas reconocidas por las empresas metalúrgicas (b).

Figure 5. Environmental formative actions carried out by industries (a) and formative needs (b).

Internet) que abren muchas posibilidades de recibir formación sin necesidad de desplazarse del puesto de trabajo.

\section{CONCLUSIONES}

A partir del estudio realizado, con datos aportados por trabajadores de PYMES del sector metalúrgico de la zona sur de la Comunidad de Madrid, pueden establecerse las siguientes conclusiones principales:

- El grado de sensibilización de las empresas metalúrgicas es limitado, siendo necesario incrementar los programas de sensibilización con participación de administraciones, universidades y asociaciones empresariales.

- Las empresas que de forma sistemática tienen implantados sistemas de gestión medioambiental, no sólo reducen su impacto, sino que obtienen beneficios empresariales como ahorro de costes y mayor satisfacción de los clientes.

- Las necesidades formativas se centran en aspectos prácticos relacionados fundamentalmente con el control de vertidos y residuos, la implantación de sistemas de gestión ambiental, así como aspectos específicos de legislación medioambiental.

- La vía preferida para recibir la información es la tele-enseñanza (Internet y cursos on-line), que evitan que el trabajador se desplace de su puesto de trabajo.
- Los resultados de este estudio muestran que, en aquellos entornos donde existe una fuerte presión normativa, como sucede en el sector de los recubrimientos, la aplicación de un sistema de gestión medioambiental es el medio más sencillo de cumplir con las exigencias de la legislación.

\section{Agradecimientos}

Los autores desean agradecer a la Fundación Biodiversidad y al Fondo Social Europeo el apoyo económico recibido para la realización de este estudio.

\section{REFERENCIAS}

[1] S.K.T. Andrews, J. Stearne y J.D. Orbell, J. Cleaner Prod. 10 (2002) 373-380.

[2] http://www.biodiversidad.urjc.es

[3] A.Ureña, J. Rams y F.J. MÉndez, Análisis sectorial de la problemática y necesidades de formación medioambientales de las PYMES en la zona sur de Madrid. Sector metalúrgico, Universidad Rey Juan Carlos.

[4] http://www.ihobe.es

[5] M.D. Escalera, E. Otero, J. Rodriguez y V. Utrilla, Guía de Buenas prácticas medioambientales. Sector metalúrgico, Universidad Rey Juan Carlos.

[6] ISO 14001. Int. Org. for Standarization, 1996.

[7] Official Journal of the European Communities. EC No. $761 / 2001$. 